\title{
An ontological approach to logistics
}

\author{
Laura Daniele* — Luís Ferreira Pires** \\ *TNO - Netherlands Organization for Applied Scientific Research \\ 2600 GB Delft \\ The Netherlands \\ laura.daniele@tno.nl \\ **University of Twente \\ 7500 AE Enschede \\ The Netherlands \\ l.ferreirapires@utwente.nl
}

\begin{abstract}
In today's global market, the competitiveness of enterprises is strongly dictated by their ability to collaborate with other enterprises. Ontologies enable common understanding of concepts and have been acknowledged as a powerful means to foster collaboration, both within the boundaries of an individual enterprise (intra-enterprise) as outside these boundaries (inter-enterprise). This paper argues that the use of ontologies can be beneficial for enterprise interoperability in the logistics domain, to improve communication and foster knowledge reuse, to facilitate the integration of existing systems and to support the development process of software solutions. Our experience shows that the development of ontologies for logistics is not a trivial task, and guidelines and best practices are necessary in this domain, especially to bridge the gap between theory and practice. On the one hand, proper theoretical and methodological support for ontology engineering is necessary in order to deliver precise, consistent and well-founded solutions to the market. On the other hand, solutions to practical issues should be provided and not take too long to be produced in order not to be detached from the original real market needs. This paper proposes an ontological approach for logistics that balances the trade-off between precision and pragmatism, by combining top-down and bottom-up practices for ontology engineering. From a top-down perspective, we promote the reuse of existing general-purpose (upper) ontologies and specialize them for the purpose of logistics. From a bottom-up perspective, we reuse code lists and classifications that already exist in logistics to support the creation of instances of our upper level concepts. The paper also presents a representative fragment of our core ontology for logistics and identifies areas for further work in ontology engineering for logistics.
\end{abstract}




\section{Introduction}

In today's global market, the competitiveness of enterprises is strongly dictated by their ability to collaborate with other enterprises. This collaboration allows enterprises to discover new opportunities or joint efforts with other enterprises in order to strengthen their competitive position (Doumeingts et al., 2007, Li 2007). Ontologies enable common understanding of concepts, and have been acknowledged as a powerful means to foster collaboration, both within the boundaries of an individual enterprise (intra-enterprise) as outside these boundaries (inter-enterprise) (Fox 1998, Grubic et al., 2007, Uschold et al., 1996). In relation to enterprise interoperability, ontologies are potentially beneficial for the following three main purposes: (i) improve communication and re-use of knowledge, by providing a shared understanding that reduces ambiguities and misunderstanding in the terminology adopted in a certain domain; (ii) facilitate the integration of existing systems, by providing a reference model that allows translation and matching, possibly automatically, among multiple heterogeneous systems that have been developed based on different semantic representations; and (iii) support the engineering process of software solutions, by providing a basis for automated specification, analysis and consistency checking of software under development.

In the past decades, logistics companies have grown individually with the goals of increasing efficiency, cutting costs and improving service offers for their customers. Currently, logistics companies still have the same goals, but with the additional aim of becoming more collaborative, especially at the inter-enterprise level. Logistics organizations should now be able to share and reuse data across other organizations (enterprises, authorities, etc.), instead of keeping proprietary data in several and, often, inconsistent versions. Therefore, not only a logistics organization may want to be able to expose its own data outside its boundaries, but also needs that the meaning of this data, or semantics, is correctly interpreted by others, otherwise the collaboration among organizations may lead to ambiguities and serious mistakes. In other words, there is a need for semantic interoperability among logistics organizations.

In this paper we argue that the use of ontologies can be beneficial for enterprise interoperability in the logistics domain. Although some efforts to apply ontologies to this domain have been reported before (Scheuermann et al., 2012), the work on ontologies for logistics is still immature (Grubic et al., 2007). This paper presents an ontological approach to logistics based on the methodology defined in (Uschold et al., 1996). Our approach proposes a core ontology that specifies the main concepts commonly used in logistics operations. This core ontology can be further extended for the purpose of specific logistics applications. The ontology presented is being developed in the context of the iCargo (www.i-cargo.eu) and CASSANDRA (www.cassandra-project.eu) projects, which are both co-funded by the European Union under the Seventh Framework Programme for ICT. 
The remainder of the paper is structured as follows: Section 2 presents some examples of semantic interoperability issues in logistics. Section 3 gives some background information on ontologies. Section 4 discusses possible applications of ontologies in the logistics domain. Section 5 presents our ontological approach for logistics. Section 6 presents a representative fragment of our core ontology for logistics. Section 7 discusses some related work in the area of ontologies. Finally, Section 8 presents our conclusions and future work.

\section{Motivation}

In order to motivate the need of an ontology for logistics, we have analysed some applications ${ }^{1}$ on the Internet that aim at integrating the offer and demand of logistics services and resources. The terminology used by these applications describes relevant objects for logistics such as, for example, the kind of goods that are transported, the possible mode of transport and the type of equipment used to facilitate the transport, among others. Table 1 shows how these concepts are represented across the different solutions we have analysed.

Table 1. Terminology used by different applications on the Internet to denote container/equipment used to transport some cargo/load

\begin{tabular}{|c|c|c|}
\hline & Container/Equipment & Cargo/Load \\
\hline $\begin{array}{l}\text { FreightCity } \\
\text { (www.freight } \\
\text { city.com) }\end{array}$ & $\begin{array}{l}\text { Container type: } \\
\text { refrigerated container, hazardous } \\
\text { cargo, special container, LCL (Less } \\
\text { than Container Load), tank } \\
\text { container, bulk cargo, breakbulk, } \\
\text { roro container }\end{array}$ & $\begin{array}{l}\text { Cargo type: } \\
\text { mechanics, precision instrument, } \\
\text { exhibitions, fresh \& live, garment, } \\
\text { primary materials, electronics, } \\
\text { chemicals, ironware, minerals, junks, } \\
\text { personal belongings, grain, steel, food }\end{array}$ \\
\hline $\begin{array}{l}\text { Transport } \\
\text { Marketplace } \\
\text { (www.transport } \\
\text { marketplace.com) }\end{array}$ & $\begin{array}{l}\text { Type: } \\
\text { container (dry, flat, HC, open top, } \\
\text { refeer), bulk liquid, bulk solid, less } \\
\text { than truckload, FCL (Full Container } \\
\text { Load), LCL (Less than Container } \\
\text { Load) }\end{array}$ & $\begin{array}{l}\text { Load: } \\
\text { general cargo, controlled temperature, } \\
\text { heavy lift, oversized, dangerous } \\
\text { (flammable gases, toxic substances, } \\
\text { flammable liquids, organic peroxides, } \\
\text { radioactive material, etc.) }\end{array}$ \\
\hline $\begin{array}{l}\text { Shipping } \\
\text { Containers24 } \\
\text { (www.shipping } \\
\text { containers24.com) }\end{array}$ & $\begin{array}{l}\text { Shipping container type: } \\
\text { dry cargo container (open top, } \\
\text { platform or flat rack, closed } \\
\text { ventilator), specific-purpose } \\
\text { container (thermal shipping } \\
\text { container or refeers, named cargo, } \\
\text { dry bulk, tank container, etc.) }\end{array}$ & $\begin{array}{l}\text { Item: } \\
\text { heavy item, bulky item, fragile item, } \\
\text { wood, heavy and difficult to manage } \\
\text { object, food, frozen goods, perishable } \\
\text { goods, cold goods, cars, other } \\
\text { vehicles, livestock, poultry, grains, } \\
\text { dry foodstuffs, chemicals, gases, } \\
\text { hazardous liquids }\end{array}$ \\
\hline $\begin{array}{l}\text { JCtrans } \\
\text { (www.jctrans.net) }\end{array}$ & $\begin{array}{l}\text { Equipment: } \\
\text { van, reefer, container, straight truck, } \\
\text { step deck, flatbed, tanker, walking } \\
\text { floor, cargo van, stretch trailer, etc. }\end{array}$ & $\begin{array}{l}\text { Load: } \\
\text { full truckload freight, less than } \\
\text { truckload freight, shipping container, } \\
\text { livestock/pets, vehicles, tanker/liquids }\end{array}$ \\
\hline
\end{tabular}

${ }^{1}$ Disclaimer: These applications were not originally devised to be interoperable, so in our comparison we do not judge them for that. This example only aims at stressing that disparity arises whenever common agreements are missing. 
Table 1 shows that the terminology used is rather domain specific and, in order to be properly interpreted, it requires some expertise (domain knowledge) from the people processing the information. Moreover, this terminology differs across the different solutions, and it is sometimes ambiguous or inconsistent (or both). This may lead to a semantic interoperability issue due to misinterpretation, creating difficulties for a potential customer who wants to make use of the services offered by multiple systems. For example, if a customer wants to transport flammable liquids and compare different solutions, what cargo/load should be selected and what type of container/equipment serves this purpose? In the current situation, the customer should use his domain knowledge in order to understand the options offered by the solutions, and should also get acquainted with the different terminology adopted in the different solutions.

The semantic interoperability issue becomes even more relevant in case automated systems are expected to interpret the information without human intervention, in case different solutions, such as the ones shown in Table 1, have to be integrated in order to automatically interoperate. For example, consider the case of information exchange between two companies, each with its own IT system built based on a certain semantic model. The first company, which provides transportation services and operates trucks, regards a truck as the transportation means used to move products from a place of origin to a final destination. The second company, which is a factory that produces trucks, regards a truck as the product that is moved from a place of origin to a final destination using a ship as transportation means. Although the two companies seem to adopt the same terminology, they do not share the same meaning, and their information exchange may lead to serious mistakes when talking about products, transport means and their mutual relationships. In this case, the so-called false agreement problem (Guarino et al., 2009, Guizzardi 2005) arises, in which the same terminology is adopted, but with different meaning.

\section{Ontologies}

The term ontology has been used in many different ways in the literature (Borst 1997, Gruber 1993, Guarino 1998, Guarino et al., 2009, Guizzardi 2005, Studer et al., 1998, Uschold et al., 1996), so in this section we characterize ontologies for the purpose of this work.

According to its original meaning in Philosophy, an ontology concerns the study of being or existence (Gruber 1993, Guarino et al., 2009), so it concerns things that exist in the real world. In our work, we use ontologies to capture mental images of the real world, the so-called conceptualizations. However, such a conceptualization has to be based on concepts, which can be instantiated for each real world situation that we may have to conceptualize. For example, a "container" can be a concept in logistics, which can be instantiated to represent specific containers used in certain 
logistics operations. Conceptualizations exist in principle in the mind of those whose produce them, but they have to be unambiguously communicated to others. Therefore, an ontology as an engineering artefact requires a language that allows the conceptualizations to be represented and communicated as concrete descriptions (specifications). This language should be suitable to represent instances of the ontology concepts and should have a formal semantics, which allows not only unambiguous interpretation but also rigorous analysis and reasoning.

Figure 1 shows that a conceptualization with respect to (a certain portion of) the real world exists a priori in one's mind and is based on a set of concepts (Guarino 1998, Guizzardi 2005). Since, we as humans need artefacts that allow us to represent things in the real world, we have to capture an abstract conceptualization that exists in the mind of users and practitioners in terms of a concrete specification that can be processed by machines. Figure 1 shows that an ontology should be explicitly represented as a specification of a conceptualization (Gruber 1993). The conceptualization underlining an ontology should be shared (Borst 1997, Studer et al., 1998), otherwise we may run in the false agreement problem mentioned above.

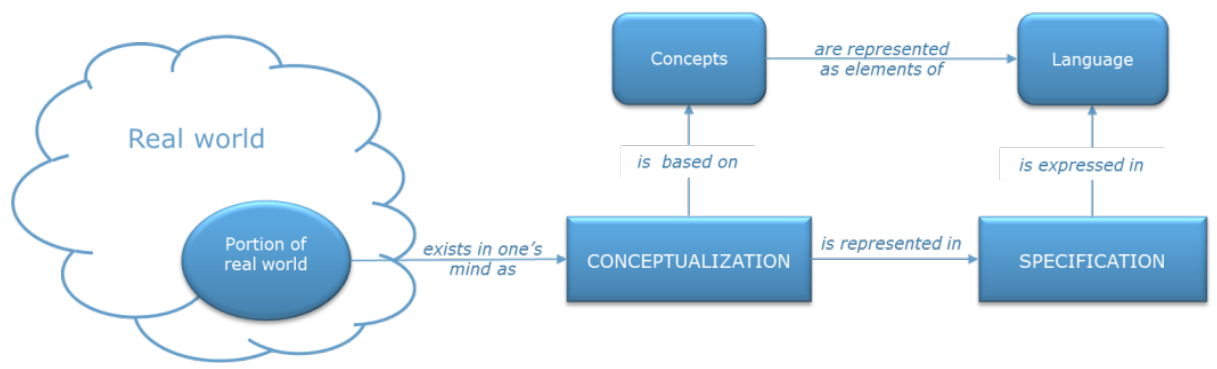

Figure 1. Ontology as explicit specification of a conceptualization

Figure 1 also shows that an ontology needs a language for its representation. More precisely, the concepts underlying a certain conceptualization need to be mapped onto elements of a suitable language. For example, the concept of a motor vehicle as a kind of object that moves on its own for the purpose of transporting goods, can be referred to by using the term truck (US English), lorry (UK English), autocarro (Italian), or vrachtwagen (Dutch). Depending on the language used to represent them, ontologies range from being highly informal, namely loosely expressed in natural language, to rigorously formal, namely strictly expressed using formal semantics, theorems and proofs (Uschold et al., 1996). Informal ontologies may lead to ambiguities, and systems that are based on such ontologies are more error-prone than systems based on formal ontologies, which, in contrast, allow automated reasoning and consistency checking. Several ontology forms are currently used in information systems and on the Internet, with varying expressiveness and complexity. These ontologies span from taxonomies of concepts related by subsumption relationships, to complete representations of concepts related by complex relationships, including axioms to constrain their intended interpretation. 
We regard a proper ontology as an engineering artefact that consists of a set of concepts and definitions used to describe a certain reality, relations among these concepts, plus a set of axioms to constrain the intended meaning of these concepts (Guarino 1998).

\section{Applications in Logistics}

We acknowledge that a single common ontology to improve communication and facilitate system integration for all possible applications in logistics is not feasible, since this ontology would get too complex and difficult to maintain. Therefore, we propose an approach of networked ontologies that is based on a core ontology, which explicitly specifies the main concepts adopted in the logistics domain. This core ontology can be further extended by creating new ontologies for the specific purpose of individual applications. The development of a core ontology (i.e. the identification of the main concepts) in logistics is not trivial task. Nevertheless, virtually all practitioners in the logistics domain informally say that "logistics is all about transporting something from a place of origin to a destination in a certain time and under certain conditions", so the key words "transport", "something", "place of origin", "destination", "time" and "conditions" are already hints to what type of concepts can be included in such a core ontology, regardless of its specific application in logistics.

\subsection{Communication purpose}

A core ontology can be used as the basis to foster communication, by facilitating interoperability among people and organizations that use different standards. Examples of standards in logistics are the following: (i) the EDIFACT standard from the United Nations, which is predominant elsewhere than North America and is based on EDI messages; and (ii) the UBL standard from OASIS, which is mostly used in Scandinavian countries and is based on XML messages. Some relevant work is based on these standards, such as, for example, the World Customs Organization (WCO) data model (www.wcoomd.org), which is aligned with the EDIFACT standard, and the Common Framework (CF) reference model (www.its.sintef9013.com/CF/v01), which is aligned with the UBL standard.

Our experience shows that each organization in logistics keeps its own standards, models and terminology. Moreover, this terminology often does not have a precise semantics, and its interpretation strongly relies on people's expertise and knowledge. Therefore, communication between people from these organizations would profit from a bottom-up process that raises the level of communication from models based on specific technical standards to an ontology that is neutral with respect to these standards. This ontology should explicitly and unambiguously specify recurring core concepts in logistics, the relationships between these concepts, and, possibly, 
mappings of these concepts to synonyms used by different standards. These mappings could be further applied in the development of tools that allow automatic translation from the core ontology to specific standard-based models. This would be beneficial also to reduce the effort of translating from one standard to another, since the ontology could be used as reference model that requires one set of mappings to each standard, instead of a dedicated set of mappings for each pair of standards (Uschold et al., 1996).

\subsection{Integration purpose}

A core ontology can be used as the basis for system integration, to facilitate the interoperability among different organizations that use various and heterogeneous IT systems with different representations of logistic concepts. Integration is especially relevant for small or medium-scale providers specialized in a specific segment of the logistic process that cannot provide door-to-door transportation services to their customer and, therefore, need to collaborate with other parties. Currently, the activity of coordinating transport providers that operate in different segments of the logistic process, such as, for example, truckers and barge operators, is supported by centralized planning systems. These systems often rely on humans that make phone calls, exchange e-mails and make use of the IT solutions offered by specific parties. However, this solution is highly dependent of the specific knowledge and expertise of people, and it is also costly and error-prone. Therefore, organizations would benefit from an integrated environment that facilitates the discovery, matching and composition of transport services, possibly by (partially) automating the error-prone activities that are currently carried out by humans.

In order to achieve this integration purpose, individual organizations could keep their own IT solutions that use different terminology for similar logistics concepts, but they should provide interfaces that specify the information necessary for interoperability. These interfaces may describe, for example, the goal and functionality of the service that they offer, the mode of transport, the area and time interval for operation, the type of resources available, i.e., the transport means and type of containers, and so forth. These interfaces should be built upon a common vocabulary, which should be derived from a common core ontology.

\subsection{Engineering purpose}

A core ontology can be used as basis for engineering purposes, by providing support for the development of software solutions, such as the Transport Management Systems (TMSs) employed in logistics for managing the transportation operations that characterize supply chains. An ontology that specifies consistently and unambiguously the resources commonly used by transport services, their conditions of usage, and the events that can possibly have an impact on these 
resources, provides a basis to develop a variety of tools. For example, analysis and simulation tools can be developed to facilitate planning and booking of the transport services and resources represented in the ontology, as well as their real-time monitoring and possibly re-planning in case of unexpected events and situations.

Another application of the core ontology for engineering purposes consists of the development of a Domain Specific Language (DSL) for logistics. Once the specific logistic domain concepts are explicitly specified in an ontology using an existing (general purpose) language, such as, for example UML and OWL DL, the same concepts can be used as building blocks to define the syntax of a dedicated language for logistics. In other words, the ontology can be used to generate a metamodel for a DSL for logistics. This DSL would allow the development of textual and (possibly) visual editors, for example, to describe logistic processes and tools to validate their correctness, as a means to facilitate supply chain management, especially for transportation operations.

\section{Approach}

In order to build an ontology that fosters semantic interoperability among logistics organizations and people, we have followed an approach based on the methodology defined in (Uschold et al., 1996) that combines top-down and bottomup practices for ontology engineering. Our ontological approach for logistics promotes reuse, modularity, extensibility, maintainability and flexibility as follows:

- Reuse of foundational concepts that are defined in existing upper ontologies (Mascardi et al., 2007) and are logistics independent, since they can be in principle used across domains. From a top-down perspective, we have specialized the upper level concepts defined in the DOLCE + DnS Ultralite ontology (www.ontologydesignpatterns.org/wiki/Ontology:DOLCE+DnS_Ultralite) for the purpose of logistics. In this way, we could provide a classification of the most relevant objects that are involved in logistics operations, such as, for example, actors, facilities, product classes, packages, pieces of equipment and transport means, and the relationships among these objects. From a bottom-up perspective, since a large amount of work has being done in logistics concerning code lists and classifications that characterize specific logistics objects, we have reused existing work so that we did not need to define from scratch classifications of transport means, packages and dangerous goods, among others.

- Modularity to allow separation and recombination of different parts of the ontology depending on specific needs, instead of creating a single common ontology for all applications in logistics, which may not be feasible, since this ontology may get too complex, and may not even be necessary. Towards this aim, we promote a network ontology approach, which is based on a logistics core ontology that specifies the main concepts commonly used in logistics operations. 
- Extensibility to allow further growth of the ontology for the purpose of specific logistics applications. For example, we have extended our core ontology with a logistics services ontology that describes the main activities and events related to logistics operations. This extension is the basis for building an environment for service discovery and composition, in which logistics providers can publish their services, and consumers can discover services that fulfil their needs. Analogously, we could extend our core ontology with a logistics documents ontology that represents all the documents exchanged in the logistics operations, a port ontology that extends the concept of port defined as a facility area in our core ontology, and so forth.

- Maintainability to facilitate the process of identifying and correcting defects, accommodate new requirements, and cope with changes in (parts of) our logistics ontology. The minimum requirement is that a new module in the network of ontologies must comply with our core ontology. This new module can further extend concepts of the core ontology and the creator of the module is responsible for its maintenance and versioning, independently from the core ontology.

- Flexibility to changes in the specific technology for ontology development, enabled by the separation of design and implementation concerns. In our approach, we separated the design of the ontology from its implementation. In the design phase, we defined the concepts of the ontology using natural language, we specified these concepts and their relations using UML class diagrams, and we defined formal axioms that capture the intended meaning of these concepts. We applied UML to represent our ontology in the design phase, since UML is a popular general-purpose language that allowed us to represent our ontology at a high abstraction level, i.e., abstracting from the ways the ontology might be implemented in actual applications. In this way we could focus on the concepts, relations and axioms that we wanted to specify, ignoring the issue of selecting the most suitable language to express them. In the implementation phase, we have specified our ontology using OWL DL, which allows automated reasoning to validate the correct use of axioms and relations, and make queries against our ontology. Due to space limitations we do not present in this paper the OWL DL version of the ontology.

\section{An Ontology for Logistics}

We consider logistics as the set of activities that take place among several actors in order to deliver certain products at the right time, right place and under the right conditions, by using suitable resources. Therefore, our logistics ontology has been built on top of some foundational (upper level) concepts, such as the following:

- Activity, which denotes some action that is relevant for the purpose of logistics and provides value for a potential customer. Activities are, for example, transport, transshipment, load, discharge, storage, consolidation and deconsolidation. These activities are atomic and can be used to compose more complex activities. 
- Actor, which represents companies, authorities or individuals that provide or request activities and operate on resources related to these activities.

- Physical Resource, which represents physical objects that are used in the logistics activities, such as, for example, the moveable resources used during the activity of transport, i.e., the transport means and equipment used to move items to their destination.

- Location, which represents the geographical area or geographical point used to define the place(s) relevant for logistics activities. Location can be coarse-grained for scheduling, since in long term planning it is sufficient to specify approximately the place of origin and destination, such as, for example, the Netherlands or the port of Rotterdam. However, location needs to be fine-grained for delivery, since one has to specify the precise address to which a certain item must be delivered.

- Time, which represents the start time, end time or time interval associated to activities. Since time is a basic (foundational) concept relevant for logistics, but common to other domains, we have re-used the representation of time proposed in the Time Ontology (http://www.w3.org/TR/owl-time), instead of specifying it from scratch.

In this paper, we cannot elaborate on all the concepts of the ontology due to space restrictions. Therefore, Figure 3 shows only an excerpt of our ontology, which focuses on the specialization of the concept of Physical Resource.

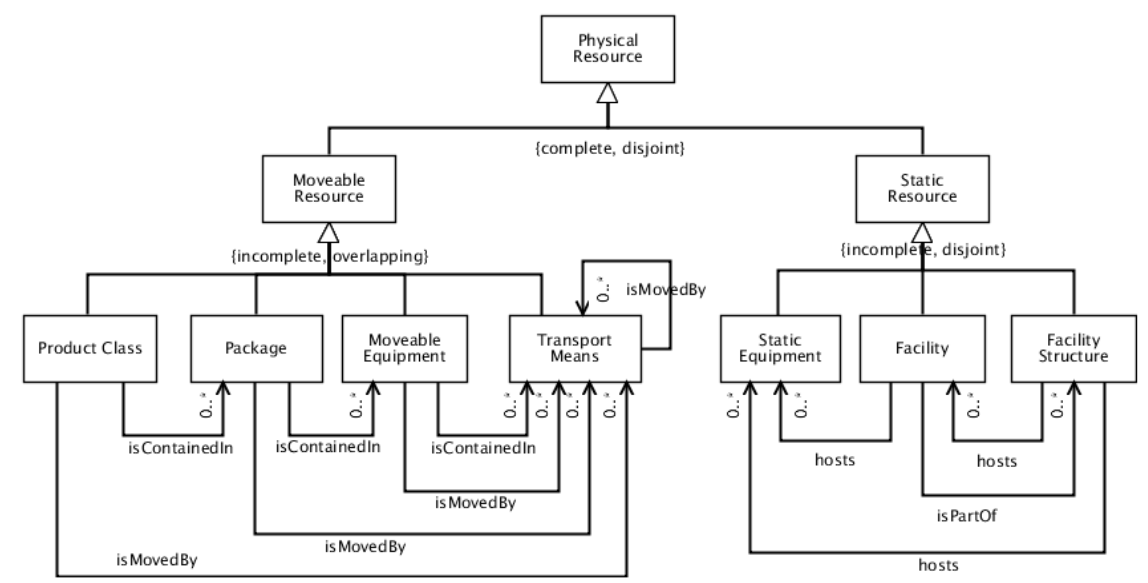

Figure 3. Core ontology for logistics focusing on the concept of Physical Resource

In Figure 3, we focus on the concept of Physical Resource, which represents tangible objects used in logistics operations, such as a piece of equipment or a facility. A Physical Resource is further specialized in a Moveable Resource, which is characterized by the capability of moving on its own or being contained for the purpose of transportation, and a Static Resource, which is used to handle moveable 
objects in a facility prior to their transportation. Table 2 clarifies and complements Figure 3.

Table 2. Definitions and properties

\begin{tabular}{|c|c|c|}
\hline & Definition & Properties \\
\hline $\begin{array}{l}\text { Product } \\
\text { class }\end{array}$ & $\begin{array}{l}\text { Object used to select proper package, moveable } \\
\text { equipment and transport means for several } \\
\text { logistics activities, especially for transport. The } \\
\text { selection is based on relevant properties of the } \\
\text { product class, such as its physical state (solid, } \\
\text { liquid, gas), required temperature, dangerousness, } \\
\text { etc. }\end{array}$ & $\begin{array}{l}\text { - Type (regular, perishable, } \\
\text { flammable, organic, toxic, heavy } \\
\text { machinery, bulk, etc.) } \\
\text { - State (solid, liquid, gas) } \\
\text { - isRefeer (Boolean value) } \\
\text { - isDangerous (Boolean value) } \\
\text { - isOversized (Boolean value) }\end{array}$ \\
\hline Package & $\begin{array}{l}\text { Material used for containment, protection and } \\
\text { movement of product classes }\end{array}$ & $\begin{array}{l}\text { - Type (carton, box, crate, barrel, } \\
\text { pallet, container, etc.) } \\
\text { - Quantity } \\
\text { - Volume }\end{array}$ \\
\hline $\begin{array}{l}\text { Moveable } \\
\text { equipment }\end{array}$ & $\begin{array}{l}\text { Reusable resource used for containment, } \\
\text { protection and movement of product classes with } \\
\text { or without package. A moveable equipment } \\
\text { cannot move on its own (unpowered vehicle), but } \\
\text { can be pulled or contained in a transport means }\end{array}$ & $\begin{array}{l}\text { - Type (container, pallet, railway } \\
\text { wagon, trailer, etc.) } \\
\text { - ID } \\
\text { - Volume } \\
\text { - Quantity }\end{array}$ \\
\hline $\begin{array}{l}\text { Transport } \\
\text { means }\end{array}$ & $\begin{array}{l}\text { Reusable resource that facilitates the activity of } \\
\text { transport and moves on its own (powered vehicle) }\end{array}$ & $\begin{array}{l}\text { - Type (aircraft, vessel, truck, } \\
\text { train) } \\
\text { - Capacity }\end{array}$ \\
\hline $\begin{array}{l}\text { Static } \\
\text { equipment }\end{array}$ & $\begin{array}{l}\text { Reusable resource that is used in a facility to } \\
\text { handle moveable resources }\end{array}$ & $\begin{array}{l}\text { - Type (crane, etc.) } \\
\text { - Facility }\end{array}$ \\
\hline Facility & $\begin{array}{l}\text { Static resource (usually a building) built, installed } \\
\text { or established to facilitate related activities in a } \\
\text { point location. A facility can be part of a facility } \\
\text { structure (for example, a terminal is part of a port) }\end{array}$ & $\begin{array}{l}\text { - Type (terminal, warehouse, } \\
\text { etc.) } \\
\text { - Location } \\
\text { - FacilityStructure }\end{array}$ \\
\hline $\begin{array}{l}\text { Facility } \\
\text { structure }\end{array}$ & $\begin{array}{l}\text { Static resource built, installed or established to } \\
\text { facilitate related activities in a geographical area. } \\
\text { A facility structure may host several facilities }\end{array}$ & $\begin{array}{l}\text { - Type (port, airport, etc.) } \\
\text { - Location (GeoArea) } \\
\text { - Facility }\end{array}$ \\
\hline
\end{tabular}

Some axioms that apply to the ontology fragment in Figure 3 are the following:

- If a moveable equipment $e$ isMoved by a transport means $t m$, then $t m$ moves $e$ (i.e., the relation moves is the inverse of isMoved);

- If a product class $p c$ isContained in a package $p$, and $p$ isContained in a moveable equipment $e$, then $p c$ isContained in $e$ (i.e., the relation isContained is transitive);

- If a product class pc is dangerous (property isDangerous is true), and $p c$ is Contained in a moveable equipment $e$, then $e$ is dangerous (property isDangerous is true). This means that property isDangerous is transitive;

- If a product class $p c$ is Contained in a package $p$, then $p$ isContained in $p c$ does not hold (i.e., the relation is Contained is asymmetric); 
- If a product class $p c$ is of type perishable, and its physical state is solid, then its property isRefeer is true and $p c$ isContained in a moveable equipment $e$, such that $e$ is a refeer container.

\section{Related Work}

The application of ontologies in logistics is still limited, however, the awareness that ontologies provide a means to foster information sharing and system interoperability and, consequently, strengthen the competitive position of enterprises, is gradually increasing. Although the work reported in (Grubic et al., 2010) targets supply chain ontologies, namely a broader domain than the logistics domain addressed in this paper, we have used some of the conclusions of (Grubic et $a l ., 2010)$ as input for our work, especially concerning the following needs: (i) combining theoretical support with empirical and field based research when creating supply chains ontologies, addressing the importance of the concept of time that is often neglected, and creating a proper ontology that is more expressive than a simple taxonomy. The ontology presented in this paper addresses the mentioned issues since it combines a top-down approach, which gives theoretical support for ontology engineering, with a bottom-up approach that considers the needs of the market and the expertise of the practitioners in the field, and explicitly addresses the concepts of time. Moreover, our ontology is not a simple taxonomy, but specifies concepts, definitions of these concepts, relations and axioms.

Although some relevant work has dealt with general-purpose ontologies that can be used across different domains (Fox et al., 1998, Madni et al., 2001, Uschold et al., 1998), we observed that these ontologies have limited applicability in logistics since they do not capture the specific issues of this domain. For example, the Enterprise Ontology of (Uschold et al., 1998) specifies concepts and relations that are relevant to business enterprises, but are too abstract to represent specific needs of the logistic domain. Furthermore, the work reported in (Fox et al., 1998) proposes a set of generic and reusable ontologies for enterprises, the so-called TOVE ontologies. Although the TOVE ontology for resources (Fadel et al., 1996) includes some concepts that can be aligned with the Moveable Resource and Static Resource concepts presented in this paper, the TOVE ontology targets a manufacturing enterprise environment that is quite different from the logistics represented in our core ontology.

\section{Conclusions}

Our experience shows that the development of ontologies for logistics is not a trivial task, and guidelines and best practices are necessary in this domain, especially to bridge the gap between theory and practice. On the one hand, proper theoretical and methodological support for ontology engineering is necessary in order to deliver precise, consistent and well-founded solutions to the market. On the other hand, 
solutions to practical issues should be provided and not take too long to be produced in order not to be detached from the original real market needs. This paper proposed an ontological approach for logistics that balances the trade-off between precision and pragmatism, offering the best of both worlds to logistics professionals and organizations. This approach prescribes the development of a network of ontologies that is based on a core ontology, which explicitly specifies the main concepts adopted in the logistics domain. This core ontology can be further extended by creating new ontologies for the specific purpose of individual applications.

Current work in the iCargo and CASSANDRA projects focuses on the realization of prototypes for specific applications that facilitate interoperability among logistics organizations. These applications are based on the ontology proposed in this paper and their realization is used to validate the concepts described in this work and to extend our logistics core ontology with new modules that become relevant during the implementation process. One of the applications consists of an environment for service discovery and composition, in which logistics service providers that use different terminology and standards can specify their services, while potential customers can discover the (composition of) services that better suit to their needs. Further work needs to be done to define mappings of our ontology to specific standards in order to: (i) validate the logistics concepts and relations that we have identified in this work, and (ii) demonstrate that our ontology can be actually used as a shared model of consensus independently of specific standards.

Acknowledgements

The authors would like to thank Wout Hofman (TNO) and Ad Schrier (TNO) for their contribution in the development of the ontology presented in this paper.

\section{References}

Borst, W., "Construction of Engineering Ontologies". PhD Thesis, University of Twente, Enschede, The Netherlands (1997)

Doumeingts, G., Muller, J.P., Morel, G., Vallespir, B., Fox, M.S., Gruninger M., Enterprise Interoperability-New Challenges and Approaches. Springer Verlag (2007)

Fadel,F.G., Fox, M.S., Gruninger, M., “A Generic Enterprise Resource Ontology”. In: Proceedings of the $3^{\text {rd }}$ IEEE Workshop on Enabling Technologies: Infrastructure for Collaborative Enterprise, pp. 117-128 (1996)

Fox, M.S., Gruninger M., “Enterprise Modeling”. In: AI Magazine, vol. 19 (3), pp. 109-121. American Association for Artificial Intelligence (AAAI) Press (1998)

Gruber, T. R., “A Translation Approach to Portable Ontology Specifications”. In: Knowledge Acquisition, vol. 5(2), pp.199-220 (1993)

Gruber, T. R., “Ontology”. In: Encyclopedia of Database Systems. Springer Verlag (2009) 
Grubic, T., Fan, I.S.: "Supply chain ontology: Review, analysis and synthesis". In: Computers in Industry, vol. 61(8), pp. 776-786. Elsevier Science Press (2010)

Guarino, N., "Formal Ontology and Information Systems". In: Proceedings of the 1st International Conference on Formal Ontology in Information Systems (FOIS), pp. 3-15. IOS Press, Amsterdam (1998)

Guarino, N., Munsen, M.A., “Applied Ontology: Focusing on Content”. In: Applied Ontology, vol.1, pp. 1-5. IOS Press (2005)

Guarino, N., Oberle, D., Staan, S., "What is an Ontology". In: Handbook on Ontologies, pp. 1-17. Springer Verlag (2009)

Guizzardi, G., "Ontological Foundations for Structural Conceptual Models". PhD Thesis, University of Twente, The Netherlands (2005)

Li, M.S., "Meeting the Grand Challenges of the Enterprise Interoperability Research Roadmap - A Business Perspective". In: Expanding the Knowledge of Economy: Issues, Applications, Case studies, pp. 5-13. IOS Press (2007)

Lin, H.K., Hardig, J.A., "A Manufacturing System Engineering Ontology Model on the Semantic Web for Inter-Enterprise Collaboration”. In: Computers in Industry, vol. 58 (5), pp. 428-437. Elsevier Science Press (2007)

Madni, A.M., Lin, W., Madni, C.C., "IDEON ${ }^{\mathrm{TM}}$ : an Extensible Ontology for Designing, Integrating and Managing Collaborative Distributed Enterprises". In: System Engineering, vol. 4 (1), pp. 35-48 (2001)

Mascardi, V., Cordì, V., Rosso, P., “A Comparison of Upper Ontologies”. Technical Report DISI-TR-06-2, Genova, Italy (2007)

Scheuermann, A., Hoxha, J., "Ontologies for Intelligent Provision of Logistics Services". In: Proceedings of the 7th International Conference on Internet and Web Applications and Services (ICIW), pp. 106-111. IARIA (2012)

Studer, R. Benjamins, R., Fensel, D., "Knowledge engineering: Principles and methods". In: Data \& Knowledge Engineering, vol. 25(1-2), pp.161-198 (1998)

Uschold, M., Gruninger, M., "Ontologies: Principles, Methods and Applications". In: the Knowledge Engineering Review, vol. 11(2), pp. 93-136. Cambridge University Press (1996)

Uschold, M., King, M., Moralee, S., Zorgios, Y., "The Enterprise Ontology". In: the Knowledge Engineering Review, vol. 13 (1), pp. 31-89. Cambridge University Press (1998) 